\title{
The Big Dipper navigation system simulation and analysis based on STK
}

\author{
Yang Bing ${ }^{\mathrm{a}}$, Li Wei Bo ${ }^{\mathrm{b}}$ \\ The Bengbu Naval Petty Office Academy, Bengbu, China, 233101;Naval Aeronautical Engineering \\ Institute, Yantai, China, 264000 \\ a174826540@qq.com, ${ }^{\mathrm{b}} 482654 w b @ 163 . c o m$
}

Keywords: Big Dipper navigation system; Satellite simulation toolkit; Constellation design; Satellite visibility; Precision dilution

\begin{abstract}
Using simulation satellite tool kit STK, combining with foreign technical experience of global navigation system and technical data released by Big Dipper navigation system at present, the paper makes simulation and analysis in detail on Big Dipper navigation system's satellite constellation design, visibility, positioning accuracy, etc. STK lifelike graphics display allows the constellation simulation of Big Dipper navigation system has good visual effect, through the analysis of satellite visibility and positioning accuracy, the results show that the Big Dipper navigation system is a satellite navigation system under global framework which has good localization performance, and can provide users with high precision navigation and positioning services. The work provides a certain reference significance as the construction and application of Big Dipper satellite navigation system.
\end{abstract}

\section{Introduction}

Big Dipper Navigation Satellite System is a global Satellite Navigation System of Chinese independent development and implementing. The system construction goal is to build an independent and open compatible, advanced technology, stable and reliable worldwide covered Big Dipper navigation system, promote satellite navigation industrial chain formation, form perfect national satellite navigation application industry support, promote and guarantee system, and promote satellite navigation wide usage in national economic and social industries.

At present, the Big Dipper navigation system is in constellation network construction stage. Around 2020, global coverage of Big Dipper navigation system will be established. Therefore, the system simulation is a prerequisite for us to carry out the follow-up work. In view of the above background, this article makes simulation and analysis in detail of the Big Dipper navigation system design, satellite constellation design visibility and positioning accuracy with the help of international famous simulation analysis platform Satellite Tool Kit (STK).

\section{Constellation design and simulation}

Currently the world's major satellite navigation system adopts Walker constellation layout. Walker constellation is composed of a set of same orbital period and dip angle of circular orbit satellites, remembered as equally spaced evenly distributed Walker T/P/F each orbit satellite, and the longitude of ascending node of the spacing between each orbit plane is evenly with the same angle, so $\mathrm{T}$ (satellite number) $=\mathrm{s}$ ( satellite number with orbit) $* \mathrm{P}$ (track number). Two adjacent orbital satellite relative phase is determined by phase parameter $\mathrm{F}$, and $\mathrm{F}$ is the"gap" number $\left(360^{\circ} / \mathrm{T}\right)$ between he Oriental satellite to the west satellite orbit. $\mathrm{F}$ is 0 to $1 \mathrm{P}$ integers.

Satellite navigation, compared with satellite communication system, has a different machine and limit on constellation. The most obvious is the need to multiple coverage (that is, need more visible satellite navigation applications at the same time). Take GPS system for example. GPS navigation calculating at least needs 4 user visible satellite to provide at least four observations for users to determine three-dimensional position and time required. Therefore, a major limitation of GPS is that 
at least four heavy covers must always be provided. To reliably ensure the levels of coverage, the actual GPS constellation design provides more than 4 heavy covers, so even if there is a satellite failure, it can maintain at least four satellites visible.

For satellite radio navigation system (RNSS system) constellation choice, the theory and practice show that Leo satellite constellation under $2000 \mathrm{~km}$ height is inappropriate. Analysis shows that Walker constellation satellite number under height of $2000 \mathrm{~km}$ is 4 times $20000 \mathrm{~km}$ Walker constellation satellite number [1]. This will lead to a surge in cost and maintain expense system. For MEO Walker constellation with the height of $20000 \mathrm{~km}$, regardless of satellite total is 24,27 or 30 , use three orbital planes with the highest availability. The European Galileo satellite navigation system conclusions and experience in constellation design as follows [2]:

A. To achieve high performance indicators, we need at least 24 satellites. Satellite altitude effects on performance indicators are weakened with the increase of satellite number. As global satellite constellation number is greater than or equal to 27 , and there is no need to consider satellite altitude's contribution to the accuracy;

B. 30 MEO satellite constellation scheme is for optimal. Choose Walker 30/3/1 constellation design for the optimal solution. When the semimajor axis is greater than or equal to $25000 \mathrm{~km}$, we all can make vertical and horizontal precision better than that of $5.5 \mathrm{~m}$ (availability is better than 99.7\%);

C. In order to further improve the availability, we should increase the backup satellite without modifying the constellation orbit.

In reference [3], Big Dipper navigation system is composed of 5 GEO satellites and 24 30 MEO satellites in space, which is located in 3 orbital planes with the orbital inclination of 55 . The running period is 12 hours and 55 minutes. It is a satellite navigation system in global framework and has good localization performance.

With all factors discussed above, choose 5GEO 30MEO constellation scheme, use STK software for constellation modeling and simulation [4]. In STK own satellite database, three Big Dipper stars Big Dipper1ABig Dipper1C navigation system GEO satellite and Big Dipper MEO satellite Dipper2A data can be found. Big Dipper2A is taken as the star seeds, get Walker 30/3/1 constellations. At the same time, considering the demands of navigation and the surrounding areas of China area, set up two GEO satellites located $\left(86^{\circ} \mathrm{E}, 0^{\circ} \mathrm{N}, 36000 \mathrm{~km}\right)$ and $\left(120^{\circ} \mathrm{E}, 0^{\circ} \mathrm{N}, 36000\right.$ $\mathrm{km})$. In order to ensure the accuracy of simulation calculation of satellite orbit, select high precision gravity potential model, atmospheric drag model (Harris Priester atmospheric model), sun light pressure model, such as using high order Runge-Kutta-Fehlberg algorithm integrating satellite motion equation [5] in the calculation model. Finally, stimulate three-dimensional simulation view of Big Dipper navigation system constellation distribution, star point trajectory under two-dimensional projection, respectively, is shown in Figure 1, in which Big Dipper1A Big Dipper1E are 5 GEO satellite, Big Dipper2A 101 Big Dipper2A 110、Big Dipper2A 201 Big Dipper2A 210 and Big Dipper2A 301 Big Dipper2A 310 are respectively corresponding to $30 \mathrm{MEO}$ satellites on three orbit planes.

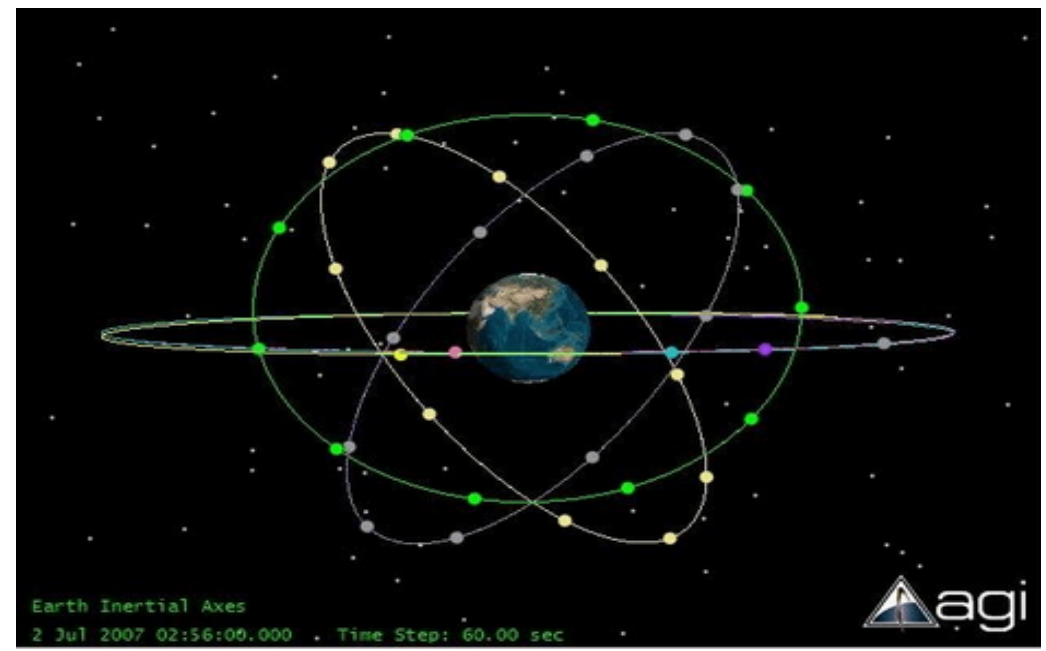

Figure 1 5GEO 30MEO satellite constellation space 


\section{Positioning accuracy analysis}

Most users concern most the location precision and credibility under given precision. Use Big Dipper navigation system for positioning. Its precision mainly depends on the following two factors: one is the geometric distribution of the tested satellite in space, which is often referred to as satellite distribution geometry; Second is the accuracy of observation. Location precision can be expressed with the following formula [7]:

Accuracy = UERE x DOP (1)

In it, Accuracy is position accuracy; UERE is equivalent distance error for the user; DOP is precision factor; the smaller its value is, the higher user location accuracy is. Equivalent distance error is the change value of pseudorange observation value predicted according to the various factors on satellite receiver path (such as clock difference, ionospheric delay, etc.), precision factor reflects the space geometric distribution of satellites, and it is a function of constellation size and orbit parameters. Usually there are plane position precision dilution HDOP, vertical precision dilution VDOP, space position precision dilution factor PDOP, receiver clock precision TDOP and geometric precision dilution GDOP. Using the above accuracy factors, we can make evaluation of positioning accuracy from different aspects.

STK coverage analysis module can be used to analyze single object or signs global and regional coverage problem. In coverage analysis, STK can not only provide detailed analysis report and charts, but cover the change of synchronous simulation, and give full consideration to all objects access restrictions to avoid calculation error.

For ground station Beijing, compute each site DOP value in simulation period, and draw its curve changes over time, which is shown in Figure 2 and Figure 3.

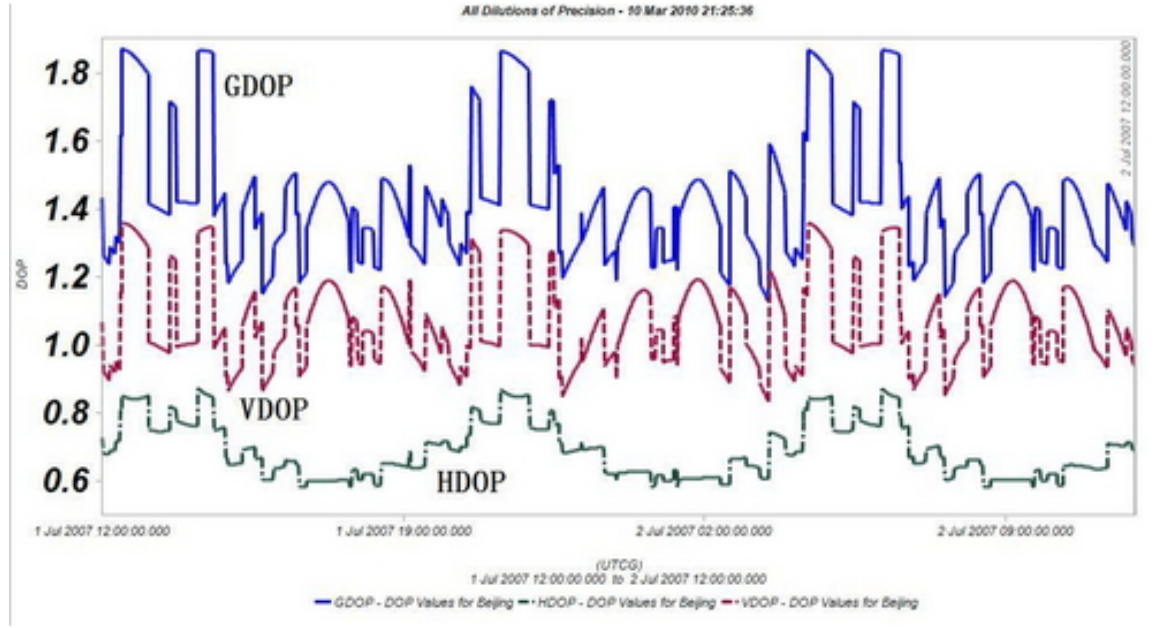

Figure 2 GDOP, VDOP and HDOP graph changes over time

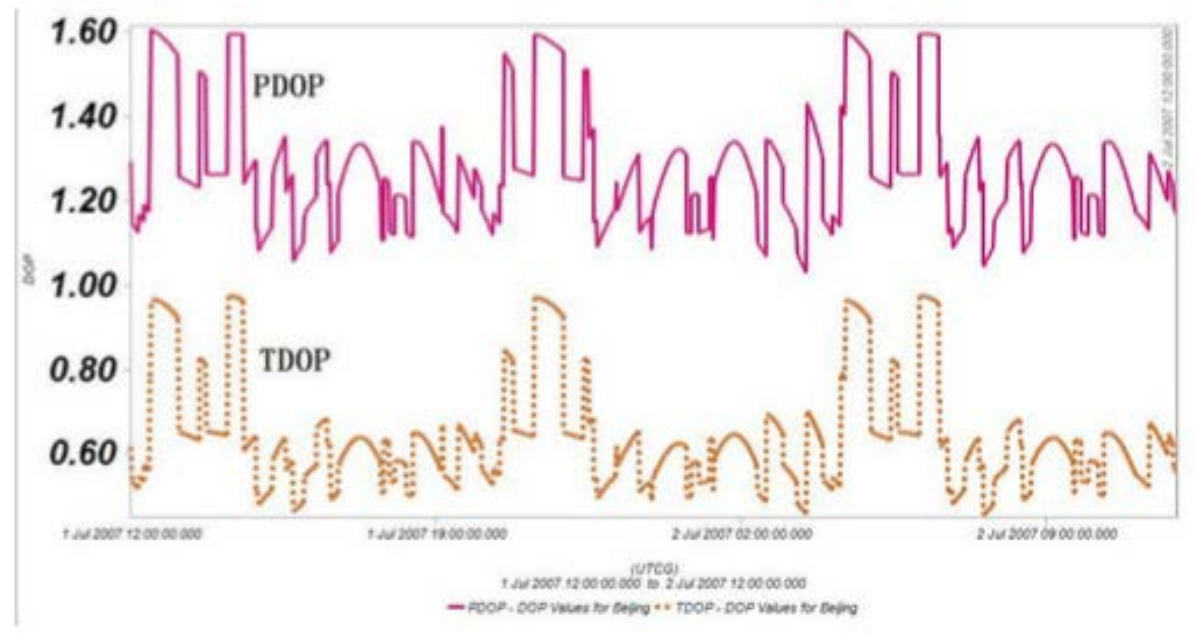

Figure 3 PDOP and TDOP graph changes over time

In addition, make covering analysis in world scope and study DOP value changing with 
geographic location space change. Spatial resolution takes $1^{\circ} \mathrm{x} 1^{\circ}$ to analyze geometric precision dilution GDOP change along with the change of latitude and longitude, which is shown in Figure 4 and 5.

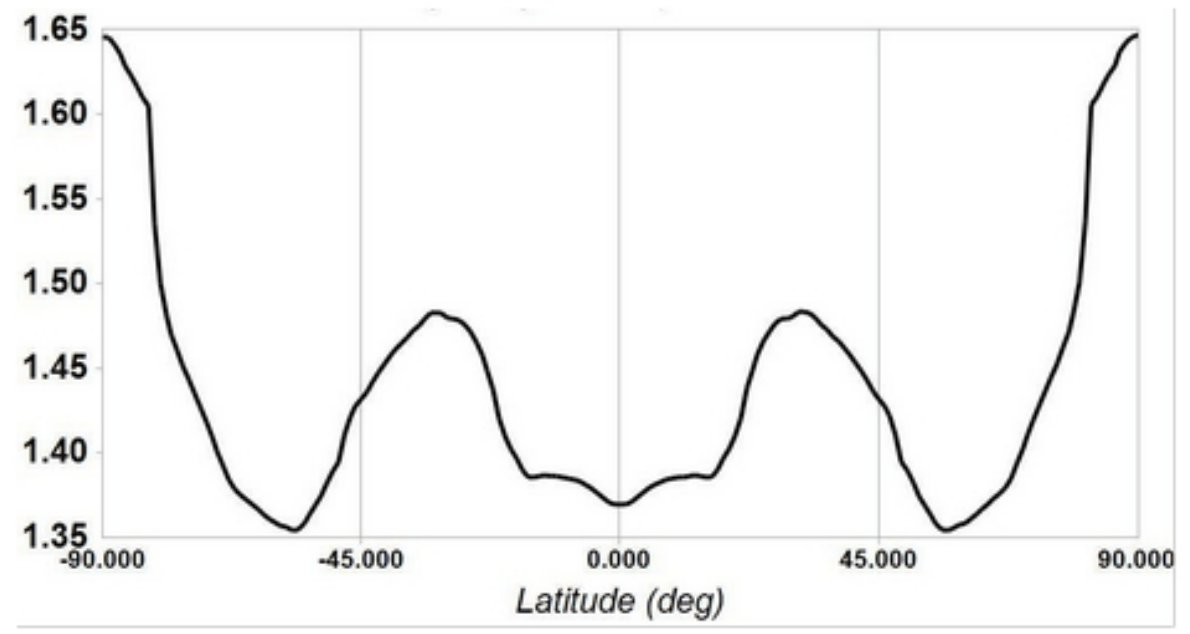

Figure 4 GDOP latitude change graph

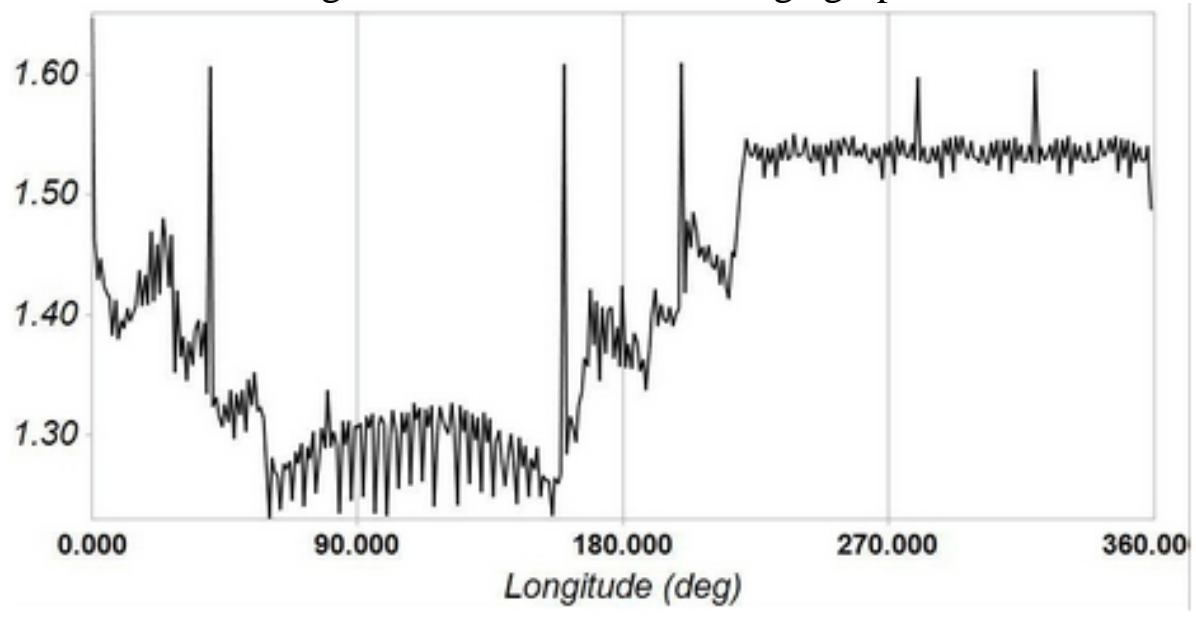

Figure 5 GDOP longitude change curve

It can be seen from the diagrams that the Big Dipper satellite navigation system GDOP value in global scope is within 1.7, and on the whole curve has less ups and downs, which indicates that Big Dipper satellite navigation system has good system continuity. GDOP value changing with longitude change is slightly more significant than with latitude direction change. In low latitudes, GDOP value is relatively small and stable, as a whole at about 1.65 . Because there are 5 GEO satellites to strengthen regional navigation performance, therefore, in our country and the surrounding areas longitude scope, GDOP is slightly smaller than other longitude range, at around 1.30. Within the scope of other longitude, GDOP levels are around 1.60.

Therefore, the analysis shows that the design of Big Dipper satellite navigation system on a global scale has good covering quality, and at the same time is a kind of satellite navigation system under global architecture with good localization performance, and can provide users with high precision navigation and positioning services.

\section{Conclusion}

STK, as an advanced satellite tool, has features such as strong computing power, lifelike graphics display, comprehensive functions analysis and reliable data report. This paper carried on the detailed simulation and analysis of Big Dipper satellite navigation system's satellite constellation design, visibility analysis and position precision and so on. Satellite constellation and star point trajectory show good intuitive visual effects. The simulation of each satellite emerging and emitting time (track) and the number of visible satellites is of great significance on related signal effectively capture work. 
From the chart report from positioning accuracy analysis can intuitively understand the Big Dipper satellite navigation system on a global scale with good covering quality, and is a satellite navigation system under global framework with good localization performance, and can provide users with high precision navigation and positioning services.

Big Dipper satellite navigation system is still at the construction stage. Based on STK, this paper carries on the simulation analysis on Big Dipper system with powerful satellite platform. The simulation accuracy has yet to be fully completed, and put into operation after further verification. The paper has a certain meaning to explore Big Dipper satellite navigation system application field, and at the same time provide the corresponding reference for specific space design.

\section{Reference}

[1]Paul Massatt, Micheal Zeitzew. The GPS Constellation Design-Current and Projected[C].Proceedings of The National

Technhttp://doc.xuehai.net/b1c164fa98a00378980807b1c.htmlical Meeting "NAVIGATION 2000".Long Beach.California,1998:569-574

[2] System level testify file. European global navigation satellite system (GNSS - 2) comparative study (8) [R]. Zhou Aosong. China Academy Space Technology. 2001

[3] Tan Shusen. The development and thinking of Big Dipper satellite navigation system [J]. Journal of Aerospace, 2008, 29 (2) : 392-396

[4] Yang Ying, Wang Qi. STK application in computer simulation [M]. Beijing, National Defense Industry Press, 2005:87-120

[5] Chai Lin, Yuan Jianping, Fang qun, etc. Constellation design and performance evaluation based on STK [J]. Journal of Aerospace, 2003, 24 (4) : 421-423

[6] Dai Mingxin, Zhang Wenming, Wang Xuesong. Design and simulation based on STK's SAR satellite orbit prediction [J]. Modern Defense Technology, 2008, 4 (1) : 5-9

[7] Zhou Guangyong, Li Liangliang. Global satellite navigation and positioning system DOP value simulation based on STK [J]. Journal of Geospatial Information, 2009, 7 (3) : 102-104 